УДК 621.315 .592

\title{
Расчет резонансных состояний кулоновских акцепторов в бесщелевых полупроводниках
}

\author{
(C) М.C. Жолудев ${ }^{1,2}$, В.В. Румянцев ${ }^{1,2}$, C.В. Морозов ${ }^{1,2}$ \\ ${ }^{1}$ Институт фризики микроструктур Российской академии наук, \\ 603950 Нижний Новгород, Россия \\ ${ }^{2}$ Нижегородский государственный университет им. Н.И. Лобачевского, \\ 603950 Нижний Новгород, Россия \\ E-mail: zholudev@ipmras
}

Поступила в Редакцию 22 декабря 2020 г.

В окончательной редакции 30 декабря 2020 г.

Принята к публикации 30 декабря 2020 г.

Проведены расчеты волновых функций электрона в зоне проводимости бесщелевого полупроводника вблизи кулоновского акцептора. Использовалась модель Латтинжера в приближении сферической симметрии. Получена зависимость энергии резонансных состояний акцептора от отношения масс электронов и дырок.

Ключевые слова: бесщелевой полупроводник, примесь, резонансные состояния, матрица рассеяния.

DOI: $10.21883 /$ FTP.2021.05.50825.9582

\section{1. Введение}

В последнее время все бо́льшую актуальность приобретает изучение примесных состояний в узкозонных полупроводниковых структурах, таких как объемные пленки и квантовые ямы на основе $\mathrm{Hg}_{1-x} \mathrm{Cd}_{x} \mathrm{Te}$ (кадмий-ртуть-теллур, КРТ). Помимо фундаментального интереса к таким системам, этому способствуют значительные успехи в получении длинноволнового стимулированного излучения в узкозонных гетероструктуpax с квантовыми ямами $\mathrm{Hg}_{1-x} \mathrm{Cd}_{x} \mathrm{Te} / \mathrm{Cd}_{y} \mathrm{Hg}_{1-y} \mathrm{Te}$ [1]. Последнее обстоятельство делает весьма актуальными задачи создания $p-n$-перехода и изучения влияния примесей на процессы безызлучательной рекомбинации в образцах такого типа.

Предыдущие работы, посвященные расчету характеристик дискретных и резонансных примесных уровней $[2-6]$, позволили существенно продвинуться в понимании природы примесно-дефектных центров в КРТ. Было показано, что большинство экспериментально наблюдаемых линий в спектрах фотопроводимости и фотолюминесценции могут быть объяснены влиянием вакансий ртути, представляющих собой двухвалентные акцепторы. Однако влиянию таких центров на непрерывный спектр (в том числе их резонансным состояниям) на данный момент уделяется мало внимания.

В работе [6] был впервые представлен расчет волновых функций и энергий резонансных состояний кулоновского акцептора в бесщелевом соединении $\mathrm{HgCdTe}$. Однако предложенный там подход имеет ограниченную область применимости из-за того, что используемые численные методы не являются устойчивыми в случае многозонных моделей. В данной работе мы предлагаем другой подход, основанный на устойчивом методе матрицы рассеяния [7]. Возможности нового метода расчета будут показаны на примере двухзонной модели бесщелевых полупроводников [8].

\section{2. Метод расчета}

При расчетах мы будем использовать модель Латтинжера в приближении сферической симметрии [8]. В рамках данной модели состояние электрона описывается тремя квантовыми числами: модулем полного углового момента $(J)$, его проекцией на ось $z(M)$ и орбитальным моментом $L=J \pm 1 / 2$. Волновая функция электрона в таком состоянии есть $[6,8,9]$

$$
\Psi_{L}^{(J, M)}(\mathbf{r})=f_{L+1}^{(J)}(r) \Phi_{L+1,3 / 2}^{(J, M)}(\mathbf{r})+f_{L-1}^{(J)}(r) \Phi_{L-1,3 / 2}^{(J, M)}(\mathbf{r}),
$$

где $\Phi_{L, 3 / 2}^{(J, M)}$ - собственные функции полного углового момента, составленные из сферических функций $Y_{L, m}(\theta, \varphi)$ и элементов базиса Кона-Латтинжера $u_{3 / 2, v}(\mathbf{r})$ с собственным моментом 3/2:

$$
\Phi_{L, 3 / 2}^{(J, M)}(\mathbf{r})=\sum_{m=-L}^{L} \sum_{v=-3 / 2}^{3 / 2} C_{L, m, 3 / 2, v}^{J, M} Y_{L, m}(\theta, \varphi) u_{3 / 2, v}(\mathbf{r}),
$$

$C_{L, m, 3 / 2, v}^{J, M}$ - коэффициенты Клебша-Гордона [10].

Таким образом, волновая функция электрона однозначно определяется парой функций $f_{L+1}^{(J)}(r)$ и $f_{L-1}^{(J)}(r)$, которые зависят только от расстояния до примесного центра, т.е. решение можно записать в виде вектора

$$
\mathbf{f}_{L}^{(J)}(r)=\left(\begin{array}{c}
f_{L+1}^{(J)}(r) \\
f_{L-1}^{(J)}(r)
\end{array}\right)
$$

В зависимости от минимального значения орбитального момента в формуле (1) состояния акцептора делятся на уровни $S$-типа, $P$-типа и т.д. [8]. 
Стационарное уравнение Шредингера для такого вектора имеет следующий вид:

$$
\left[\hat{\mathbf{H}}_{0}^{(J, L)}+V(r)\right] \mathbf{f}_{L}^{(J)}=E \mathbf{f}_{L}^{(J)} .
$$

Гамильтониан однородного полупроводника в сферически симметричной модели Латтинжера $[6,8,9]$ равен

$$
\hat{\mathbf{H}}_{0}^{(J, L)}=\left(\begin{array}{cc}
\mu_{+}^{(J, L)} \hat{K}_{-}^{(L+2)} \hat{K}_{+}^{(L+1)} & \mu_{2}^{(J, L)} \hat{K}_{+}^{(L)} \hat{K}_{+}^{(L-1)} \\
\mu_{2}^{(J, L)} \hat{K}_{-}^{(L)} \hat{K}_{-}^{(L+1)} & \mu_{-}^{(J, L)} \hat{K}_{-}^{(L)} \hat{K}_{+}^{(L-1)}
\end{array}\right),
$$

где

$$
\begin{gathered}
\hat{K}_{+}^{(L)}=-i\left(\frac{d}{d r}-\frac{L}{r}\right), \\
\hat{K}_{-}^{(L)}=i\left(\frac{d}{d r}+\frac{L+1}{r}\right), \\
\mu_{+}^{(J, L)}=1+\mu w^{(J, L)}, \\
\mu_{-}^{(J, L)}=1-\mu w^{(J, L)}, \\
\mu_{2}^{(J, L)}=\mu w_{2}^{(J, L)}, \\
w^{(J, J-1 / 2)}=-\frac{2 J-3}{4 J}, \\
w^{(J, J+1 / 2)}=\frac{2 J+5}{4(J+1)}, \\
w_{2}^{(J, J-1 / 2)}=\frac{\sqrt{3(2 J-1)(2 J+3)}}{4 J}, \\
w_{2}^{(J, J+1 / 2)}=\frac{\sqrt{3(2 J-1)(2 J+3)}}{4(J+1)} .
\end{gathered}
$$

Здесь в качестве единиц измерения длины и энергии используются соответственно эффективный боровский радиус и эффективный Ридберг [8]:

$$
\begin{gathered}
a_{0}=\frac{\hbar^{2} \varepsilon_{0} \gamma_{1}}{m_{0} e^{2}}, \\
E_{0}=\frac{m_{0} e^{4}}{2 \hbar^{2} \varepsilon_{0}^{2} \gamma_{1}} .
\end{gathered}
$$

Поэтому потенциал кулоновского центра в уравнении (2) равен

$$
V(r)=-2 \frac{Z}{r}
$$

где $Z$ - зарядовое число примесного центра.

Система из двух уравнений второго порядка (2) имеет 4 линейно независимых частных решения. Нам нужно найти такую их линейную комбинацию, для которой волновая функция будет конечной при $r=0$ и $r \rightarrow \infty$. Поскольку потенциал (3) имеет особенность в нуле, для вычисления функций (1) при малых значениях $r$ лучше всего подходит обобщенный метод Фробениуса [11]. Решение уравнений при больших $r$ можно найти методом Рунге-Кутта, однако из-за присутствия валентной зоны частные решения будут экспоненциально расти, т.е. данный метод будет неустойчивым. Для решения этой проблемы можно использовать метод стрельбы, как это было сделано в работе [6], однако такой подход не очень эффективен с точки зрения времени расчета и очень сложен для реализации в случае большого количества зон. В данной работе мы предлагаем использовать метод матрицы рассеяния [7,12-14], который обладает численной устойчивостью.

Выберем близкую к нулю точку $r_{0}$, такую, что при $r \leq r_{0}$ неустойчивость не мешает получить решение уравнения (2) с помощью последовательного применения обобщенного метода Фробениуса и метода Рунге-Кутта. Выберем также точку $r_{N}$, достаточно далекую от нуля, чтобы при $r \geq r_{N}$ мы могли считать потенциал примеси равным нулю. В области $\left[r_{0}, r_{N}\right]$ мы будем искать решение уравнения (2) с помощью метода матрицы рассеяния. Для этого разобьем ее на отрезки серией точек $r_{0}, r_{1}, \ldots, r_{N}$ и на каждом отрезке $\left[r_{j}, r_{j+1}\right]$ будем считать потенциал постоянным и равным

$$
V_{j}=\frac{V\left(r_{j}\right)+V\left(r_{j+1}\right)}{2} .
$$

Тогда уравнение (2) примет вид

$$
\hat{\mathbf{H}}_{0}^{(J, L)} \mathbf{f}_{j}=\left(E-V_{j}\right) \mathbf{f}_{j} .
$$

Уравнение (4) фактически описывает движение электрона в однородном полупроводнике. Его решениями будут комбинации из сферических функций Бесселя:

$$
\mathbf{f}_{j, n}^{(a, b)}(r)=\left(\begin{array}{l}
c_{j, n, 1} s_{L+1, \pm k_{j, n}}(r) \\
c_{j, n, 2} s_{L-1, \pm k_{j, n}}(r)
\end{array}\right) ; n=1,2,
$$

где $s_{L, k}(r)=j_{L}(k r)+i y_{L}(k r)$, а $j_{L}$ и $y_{L}-$ сферические функции Бесселя 1-го и 2-го рода соответственно. Отметим, что эти функции определены как для вещественных, так и для мнимых значений $k_{j, n}$ (см., например, [15]). Мы можем считать, что в выражении (5) $k_{j, n}$ является положительным вещественным числом или мнимым числом с положительной мнимой частью. Тогда функции $\mathbf{f}_{j, n}^{(a)}(r)$ будут сферическими волнами, бегущими от центра (для вещественных $k_{j, n}$ ), либо экспоненциально затухающими решениями (для мнимых $k_{j, n}$ ). В то же время функции $\mathbf{f}_{j, n}^{(b)}(r)$, имеющие противоположный знак волнового числа, будут соответственно сферическими волнами, бегущими к центру, либо экспоненциально растущими решениями. Такая классификация решений обеспечивает устойчивость метода матрицы рассеяния [7].

Подставив выражение (5) в уравнение (4), мы получим уравнение для волновых чисел $k_{j, n}$ и коэффициентов $c_{j, n, L}$ :

$$
\left(\begin{array}{cc}
-\mu_{+} k_{j, n}^{2}+V_{j}-E & -\mu_{2} k_{j, n}^{2} \\
-\mu_{2} k_{j, n}^{2} & -\mu_{-} k_{j, n}^{2}+V_{j}-E
\end{array}\right)\left(\begin{array}{c}
c_{j, n, L+1} \\
c_{j, n, L-1}
\end{array}\right)=0 .
$$

В случае бесщелевого полупроводника решения $k_{j, n}^{2}$ будут иметь противоположные знаки, поскольку ветви одной зоны направлены вверх, а другой - вниз. Далее для определенности мы будем считать, что $k_{j, 1}^{2}>0$, a $k_{j, 2}^{2}<0$. 
Приближенное решение уравнения (2) на отрезке $\left[r_{j}, r_{j+1}\right]$ будет линейной комбинацией всех частных решений (5):

$$
\mathbf{f}_{j}(r)=\mathbf{F}_{j}^{(a)}(r) \mathbf{a}_{j}+\mathbf{F}_{j}^{(b)}(r) \mathbf{b}_{j}
$$

где

$$
\mathbf{F}_{j}^{(a, b)}(r)=\left(\begin{array}{ll}
c_{j, 1, L+1} s_{L+1, \pm k_{j, 1}}(r) & c_{j, 2, L+1} s_{L+1, \pm k_{j, 2}}(r) \\
c_{j, 1, L-1} s_{L-1, \pm k_{j, 1}}(r) & c_{j, 2, L-1} s_{L-1, \pm k_{j, 2}}(r)
\end{array}\right) .
$$

Неизвестные коэффициенты $\mathbf{a}_{j}$ и $\mathbf{b}_{j}$ должны обеспечить непрерывность функции $\mathbf{f}_{j}(r)$ и ее первой производной на границах отрезков. Из этого условия мы можем найти выражения для трансфер-матриц, связывающих значения этих коэффициентов на соседних отрезках:

$$
\left(\begin{array}{l}
\mathbf{a}_{j} \\
\mathbf{b}_{j}
\end{array}\right)=\left(\begin{array}{ll}
\mathbf{T}_{j}^{(a a)} & \mathbf{T}_{j}^{(a b)} \\
\mathbf{T}_{j}^{(b a)} & \mathbf{T}_{j}^{(b b)}
\end{array}\right)\left(\begin{array}{l}
\mathbf{a}_{j-1} \\
\mathbf{b}_{j-1}
\end{array}\right),
$$

где

$$
\begin{aligned}
\left(\begin{array}{cc}
\mathbf{T}_{j}^{(a a)} & \mathbf{T}_{j}^{(a b)} \\
\mathbf{T}_{j}^{(b a)} & \mathbf{T}_{j}^{(b b)}
\end{array}\right)= & \left(\begin{array}{ll}
\mathbf{F}_{j}^{(a)}\left(r_{j}\right) & \mathbf{F}_{j}^{(b)}\left(r_{j}\right) \\
\mathbf{F}_{j}^{(a)}\left(r_{j}\right) & \mathbf{F}_{j}^{\prime(b)}\left(r_{j}\right)
\end{array}\right)^{-1} \\
& \times\left(\begin{array}{ll}
\mathbf{F}_{j-1}^{(a)}\left(r_{j}\right) & \mathbf{F}_{j-1}^{(b)}\left(r_{j}\right) \\
\mathbf{F}_{j-1}^{(a)}\left(r_{j}\right) & \mathbf{F}_{j-1}^{\prime(b)}\left(r_{j}\right)
\end{array}\right),
\end{aligned}
$$

а штрихи в последнем выражении означают производные по $r$.

По определению, матрица рассеяния для области $\left[r_{j_{1}}, r_{j_{2}}\right]$ удовлетворяет соотношению

$$
\left(\begin{array}{l}
\mathbf{a}_{j_{2}} \\
\mathbf{b}_{j_{1}}
\end{array}\right)=\left(\begin{array}{ll}
\mathbf{S}_{j_{1}, j_{2}}^{(a a)} & \mathbf{S}_{j_{1}, j_{2}}^{(a b)} \\
\mathbf{S}_{j_{1}, j_{2}}^{(b a)} & \mathbf{S}_{j_{1}, j_{2}}^{(b b)}
\end{array}\right)\left(\begin{array}{l}
\mathbf{a}_{j_{1}} \\
\mathbf{b}_{j_{2}}
\end{array}\right) .
$$

Метод вычисления матриц рассеяния изложен в работе [7]. Здесь мы приведем только результат:

$$
\begin{gathered}
\left(\begin{array}{ll}
\mathbf{S}_{0,0}^{(a a)} & \mathbf{S}_{0,0}^{(a b)} \\
\mathbf{S}_{0,0}^{(b a)} & \mathbf{S}_{0,0}^{(b b)}
\end{array}\right)=\left(\begin{array}{ll}
\mathbf{S}_{N, N}^{(a a)} & \mathbf{S}_{N, N}^{(a b)} \\
\mathbf{S}_{N, N}^{(b a)} & \mathbf{S}_{N, N}^{(b b)}
\end{array}\right)=\left(\begin{array}{ll}
1 & 0 \\
0 & 1
\end{array}\right), \\
\mathbf{X}_{j}=\left(\mathbf{T}_{j}^{(b b)}\right)^{-1}\left(\mathbf{T}_{j}^{(b a)} \mathbf{S}_{0, j-1}^{(a b)}\left(T_{j}^{(b b)}\right)^{-1}+1\right)^{-1} \\
\mathbf{S}_{0, j}^{(a b)}=\left(\mathbf{T}_{j}^{(a a)} \mathbf{S}_{0, j-1}^{(a b)}+\mathbf{T}_{j}^{(a b)}\right) \mathbf{X}_{j} \\
\mathbf{S}_{0, j}^{(b b)}=\mathbf{S}_{0, j-1}^{(b b)} \mathbf{X}_{j} \\
\mathbf{S}_{0, j}^{(a a)}=\left(\mathbf{T}_{j}^{(a a)}-\mathbf{S}_{0, j}^{(a b)} \mathbf{T}_{j}^{(b a)}\right) \mathbf{S}_{0, j-1}^{(a a)} \\
\mathbf{S}_{0, j}^{(b a)}=\mathbf{S}_{0, j-1}^{(b a)}-\mathbf{S}_{0, j}^{(b b)} \mathbf{T}_{j}^{(b a)} \mathbf{S}_{0, j-1}^{(a a)} \\
\tilde{\mathbf{X}}_{j}=\left(1-\left(\mathbf{T}_{j}^{(b b)}\right)^{-1} \mathbf{S}_{j, N}^{(b a)} \mathbf{T}_{j}^{(a b)}\right)^{-1}\left(\mathbf{T}_{j}^{(b b)}\right)^{-1} \\
\mathbf{S}_{j-1, N}^{(b a)}=\tilde{\mathbf{X}}_{j}\left(\mathbf{S}_{j, N}^{(b a)} \mathbf{T}_{j}^{(a a)}-\mathbf{T}_{j}^{(b a)}\right)
\end{gathered}
$$

$$
\begin{gathered}
\mathbf{S}_{j-1, N}^{(b b)}=\tilde{\mathbf{X}}_{j} \mathbf{S}_{j, N}^{(b b)}, \\
\mathbf{S}_{j-1, N}^{(a a)}=\mathbf{S}_{j, N}^{(a a)}\left(\mathbf{T}_{j}^{(a a)}-\mathbf{T}_{j}^{(b b)} \mathbf{S}_{j-1, N}^{(b a)}\right), \\
\mathbf{S}_{j-1, N}^{(a b)}=\mathbf{S}_{j, N}^{(a b)}-\mathbf{S}_{j, N}^{(a a)} \mathbf{T}_{j}^{(a b)} \mathbf{S}_{j-1, N}^{(b b)} .
\end{gathered}
$$

Коэффициенты $\mathbf{a}_{j}$ и $\mathbf{b}_{j}$ вычисляются с помощью следующих выражений [7]:

$$
\begin{aligned}
& \mathbf{a}_{j}=\left(1-\mathbf{S}_{0, j}^{(a b)} \mathbf{S}_{j, N}^{(b a)}\right)^{-1}\left(\mathbf{S}_{0, j}^{(a a)} \mathbf{a}_{0}+\mathbf{S}_{0, j}^{(a b)} \mathbf{S}_{j, N}^{(b b)} \mathbf{b}_{N}\right), \\
& \mathbf{b}_{j}=\left(1-\mathbf{S}_{j, N}^{(b a)} \mathbf{S}_{0, j}^{(a b)}\right)^{-1}\left(\mathbf{S}_{j, N}^{(b a)} \mathbf{S}_{0, j}^{(a a)} \mathbf{a}_{0}+\mathbf{S}_{j, N}^{(b b)} \mathbf{b}_{N}\right) .
\end{aligned}
$$

Чтобы воспользоваться этими формулами, как очевидно, нам потребуются значения $\mathbf{a}_{0}$ и $\mathbf{b}_{N}$. Из условия конечности волновой функции при $r \rightarrow \infty$ следует, что все компоненты вектора $\mathbf{b}_{N}$, соответствующие растущим экспонентам, должны быть равны нулю. Поскольку мы считаем, что $k_{j, 2}^{2}<0$, этот вектор равен

$$
\mathbf{b}_{N}=\left(\begin{array}{c}
b_{N, 1} \\
0
\end{array}\right) \text {. }
$$

Значение коэффициента $b_{N, 1}$ мы найдем из условия нормировки:

$$
\int_{0}^{\pi} \sin \theta d \theta \int_{0}^{2 \pi} d \varphi \int_{0}^{\infty} r^{2} d r\left[\Psi_{L, E}^{(J, M)}\right]^{*} \Psi_{L, E^{\prime}}^{(J, M)}=\delta\left(E-E^{\prime}\right) .
$$

Для вычисления вектора $\mathbf{a}_{0}$ сначала нужно с помощью обобщенного метода Фробениуса и метода Рунге-Кутта найти в области $r<r_{0}$ два линейно независимых решения уравнения (2), которые не имеют особенностей в нуле. Эти решения $\mathbf{f}_{-1, n}(r)$ имеют вид (1) и образуют квадратную матрицу

$$
\mathbf{F}_{-1}(r)=\left(\begin{array}{ll}
f_{-1,1, L+1}(r) & f_{-1,2, L+1}(r) \\
f_{-1,1, L-1}(r) & f_{-1,2, L-1}(r)
\end{array}\right),
$$

где используется индекс -1 , поскольку мы рассматриваем область слева от отрезка с индексом 0. Тогда искомое решение на отрезке $\left[0, r_{0}\right]$ будет равно

$$
\mathbf{f}_{-1}(r)=\mathbf{F}_{-1}(r) \mathbf{a}_{-1},
$$

где неизвестный вектор $\mathbf{a}_{-1}$ должен обеспечить выполнение условий непрерывности функции $\mathbf{f}(r)$ и ее первой производной в точке $r_{0}$ :

$$
\left(\begin{array}{l}
\mathbf{F}_{-1}\left(r_{j}\right) \\
\mathbf{F}_{-1}^{\prime}\left(r_{j}\right)
\end{array}\right) \mathbf{a}_{-1}=\left(\begin{array}{cc}
\mathbf{F}_{0}^{(a)}\left(r_{j}\right) & \mathbf{F}_{0}^{(b)}\left(r_{j}\right) \\
\mathbf{F}_{0}^{\prime(a)}\left(r_{j}\right) & \mathbf{F}_{0}^{(b)}\left(r_{j}\right)
\end{array}\right)\left(\begin{array}{l}
\mathbf{a}_{0} \\
\mathbf{b}_{0}
\end{array}\right)
$$

или

$$
\left(\begin{array}{c}
\mathbf{a}_{0} \\
\mathbf{b}_{0}
\end{array}\right)=\left(\begin{array}{c}
\mathbf{T}_{0}^{(a)} \\
\mathbf{T}_{0}^{(b)}
\end{array}\right) \mathbf{a}_{-1}
$$




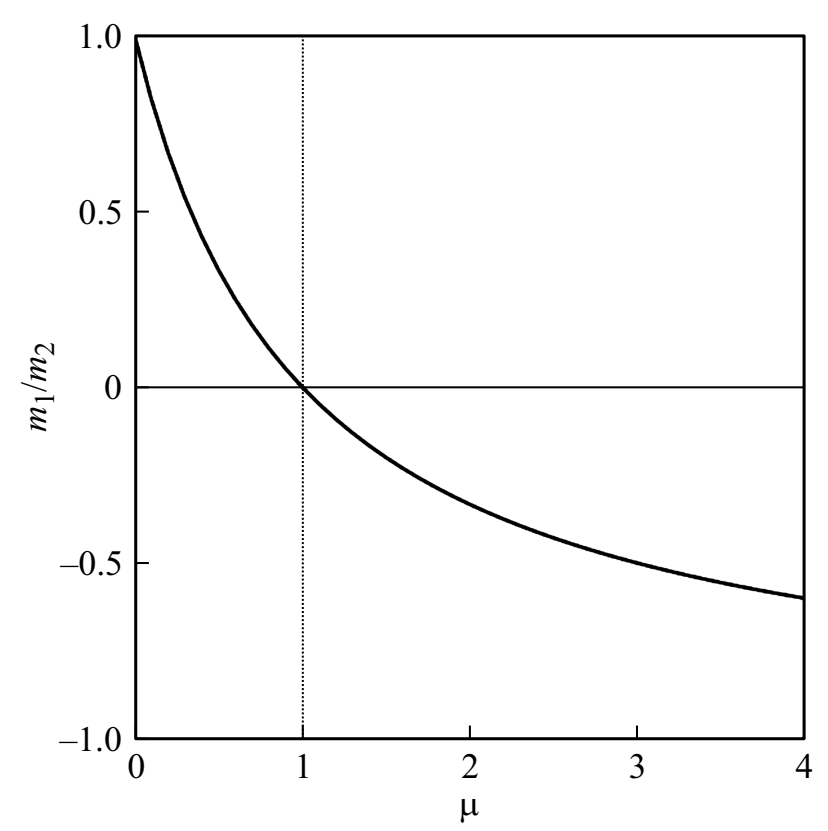

Рис. 1. Зависимость отношения эффективных масс в модели Латтинжера от параметра $\mu$.

где

$$
\left(\begin{array}{c}
\mathbf{T}_{0}^{(a)} \\
\mathbf{T}_{0}^{(b)}
\end{array}\right)=\left(\begin{array}{ll}
\mathbf{F}_{0}^{(a)}\left(r_{j}\right) & \mathbf{F}_{0}^{(b)}\left(r_{j}\right) \\
\mathbf{F}_{0}^{(a)}\left(r_{j}\right) & \mathbf{F}_{0}^{(b)}\left(r_{j}\right)
\end{array}\right)\left(\begin{array}{l}
\mathbf{F}_{-1}\left(r_{j}\right) \\
\mathbf{F}_{-1}^{\prime}\left(r_{j}\right)
\end{array}\right) .
$$

Подставив $j_{1}=0$ и $j_{2}=N$ в (7), и с учетом выражения (9) мы получим

$$
\mathbf{a}_{-1}=\left(\mathbf{T}_{0}^{(b)}-\mathbf{S}_{0, N}^{(b a)} \mathbf{T}_{0}^{(a)}\right)^{-1} \mathbf{S}_{0, N}^{(b b)} \mathbf{b}_{N}
$$

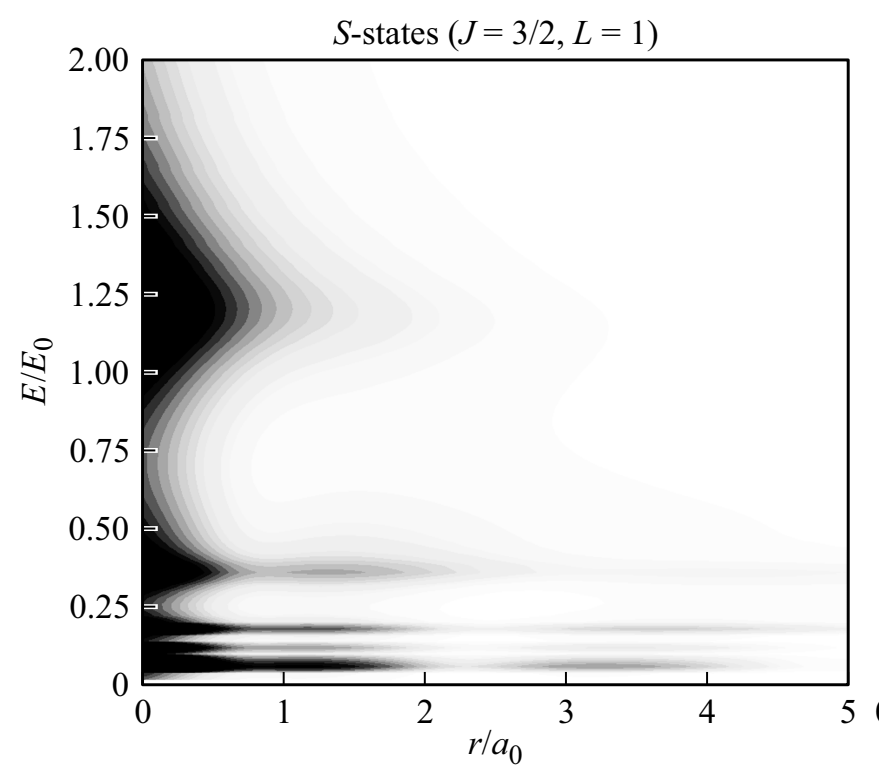

Откуда, воспользовавшись формулой (9), получаем

$$
\mathbf{a}_{0}=\mathbf{T}_{0}^{(a)}\left(\mathbf{T}_{0}^{(b)}-\mathbf{S}_{0, N}^{(b a)} \mathbf{T}_{0}^{(a)}\right)^{-1} \mathbf{S}_{0, N}^{(b b)} \mathbf{b}_{N}
$$

Таким образом, нам известны решения $\mathbf{f}_{-1, n}(r)$ и $\mathbf{f}_{j, n}^{(a, b)}(r)$, а также коэффициенты $\mathbf{a}_{-1}, \mathbf{a}_{j}$ и $\mathbf{b}_{j}$, где $j=0, \ldots, N$. Это позволяет вычислить функцию $\mathbf{f}(r)$ для любого $r$ с помощью формул (6) и (8).

\section{3. Результаты и обсуждение}

Главным параметром модели является величина

$$
\mu=\frac{4 \gamma_{2}+6 \gamma_{3}}{5 \gamma_{1}}
$$

которая определяется отношением эффективных масс в двух зонах:

$$
m_{1,2}=\frac{m_{0}}{1 \pm \mu}
$$

Для бесщелевого полупроводника одна из масс должна быть отрицательной, и это условие выполняется при $\mu>1$. Однако из рис. 1 хорошо видно, что в рамках данной модели масса в валентной зоне будет всегда меньше, чем в зоне проводимости. Нам же интересен обратный случай, так как именно он реализуется в соединении $\mathrm{HgTe}$. Для того чтобы описать такую систему, мы, воспользовавшись симметрией двухзонной модели, рассмотрим донор $(Z=1)$ вместо акцептора $(Z=-1)$ и изменим направление оси энергий на противоположное. Таким образом, в новой системе координат эффективная масса носителей в валентной зоне будет больше, чем в зоне проводимости.

Для того чтобы определить положение резонансных уровней, мы провели расчеты волновых функций в

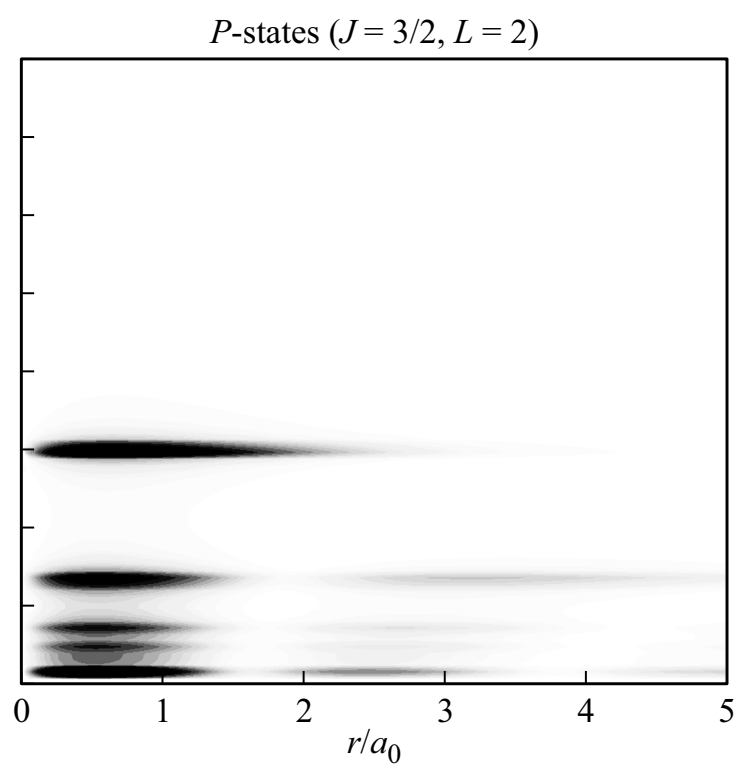

Рис. 2. Усредненная по углам зависимость плотности вероятности обнаружения электрона от его энергии $(E)$ и расстояния до примесного центра $(r)$. 


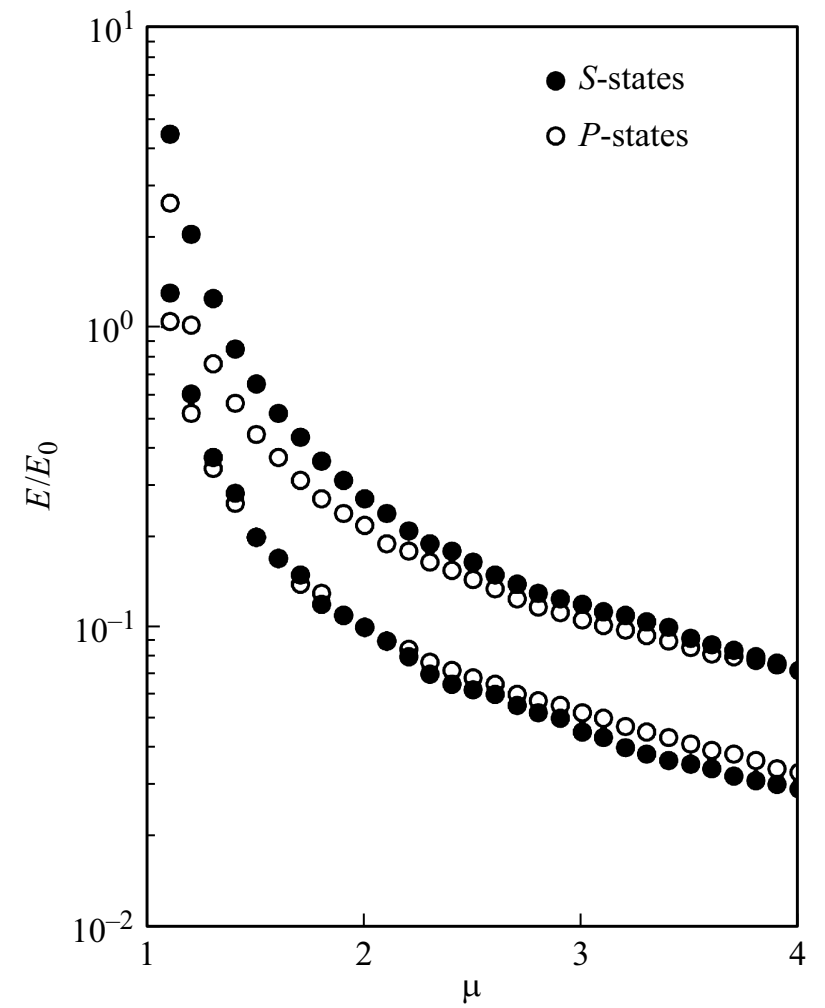

Рис. 3. Зависимость рассчитанной энергии наиболее глубоких резонансных состояний от параметра $\mu$.

зоне проводимости в широком диапазоне энергий при различных значениях параметра $\mu$. Пример полученных таким образом результатов представлен на рис. 2. Резонансные состояния хорошо видны как максимумы плотности вероятности при определенных значениях энергии. Зависимость энергии резонансных состояний от параметра $\mu$ приведена на рис. 3.

\section{4. Заключение}

Таким образом, нами разработан устойчивый метод расчета волновых функций непрерывного спектра в бесщелевых полупроводниках с учетом потенциала примеси. Высокая эффективность и устойчивость данного метода делает его весьма перспективным для численного моделирования резонансных состояний примесей в узкозонных гетероструктурах на основе соединений $\mathrm{Hg}_{1-x} \mathrm{Cd}_{x} \mathrm{Te}$.

\section{Финансирование работы}

Разработка программного обеспечения для расчета волновых функций электрона в многозонных моделях выполнена при поддержке Российского научного фонда (грант № 19-72-00128). Расчеты волновых функций в модели Латтинжера и определение энергий резонансных состояний выполнены при поддержке Министерства образования и науки Российской Федерации (проект МК-1430.2020.2).

\section{Конфликт интересов}

Авторы заявляют, что у них нет конфликта интересов.

\section{Список литературы}

[1] S. Morozov, V. Rumyantsev, M. Fadeev, M. Zholudev, K. Kudryavtsev, A. Antonov, A. Kadykov, A. Dubinov, N. Mikhailov, S. Dvoretskii, V. Gavrilenko. Appl. Phys. Lett., 111, 192101 (2017).

[2] Д.В. Козлов, В.В. Румянцев, С.В. Морозов, А.М. Кадыков, М.А. Фадеев, В.С. Варавин, Н.Н. Михайлов, С.А. Дворецкий, В.И. Гавриленко, F. Терре. ФТП, 50, 1690 (2016).

[3] V.V. Rumyantsev, D.V. Kozlov, S.V. Morozov, M.A. Fadeev, A.M. Kadykov, F. Teppe, V.S. Varavin, M.V. Yakushev, N.N. Mikhailov, S.A. Dvoretskii, V.I. Gavrilenko. Semicond. Sci. Technol., 32, 095007 (2017).

[4] Д.В. Козлов, В.В. Румянцев, С.В. Морозов, А.М. Кадыков, M.A. Фадеев, H.-W. Hubers, В.И. Гавриленко. ФТП, 52, 1257 (2018).

[5] Д.В. Козлов, В.В. Румянцев, А.М. Кадыков, М.А. Фадеев, Н.С. Куликов, В.В. Уточкин, Н.Н. Михайлов, С.А. Дворецкий, В.И. Гавриленко, Х.-В. Хюберс, Ф. Теппе, С.В. Морозов. Письма ЖЭТФ, 109, 679 (2019).

[6] М.С. Жолудев, Д.В. Козлов, Н.С. Куликов, А.А. Разова, В.И. Гавриленко, С. В. Морозов. ФТП, 54, 695 (2020).

[7] D. Yuk Kei Ko, J.C. Inkson. Phys. Rev. B, 38, 9945 (1988).

[8] A. Baldereschi, N.O. Lipari. Phys. Rev. B, 8, 2697 (1973).

[9] E.P. Pokatilov, V.A. Fonoberov, V.M. Fomin, J.T. Devreese. Phys. Rev. B, 64, 245328 (2001).

[10] Д.А. Варшалович, А.Н. Москалев, В.К. Херсонский. Квантовая теория углового момента (Л., Наука, 1975).

[11] M. Barkatou, T. Cluzeau, C. El Bacha. In: Proc. of Mathematical Theory of Networks and Systems (Budapest, Hungary, 2010) p. 1059.

[12] M.S. De Bianchi, M.D. Ventra. Eur. J. Phys., 16, 260 (1995).

[13] C. Ramirez, R. Leon. J. Phys. Soc. Jpn., 86, 114002 (2017).

[14] C. Ramirez, F.H. Gonzalez, C.G. Galvan. J. Phys. Soc. Jpn., 88, 094002 (2019).

[15] М. Абрамовиц, И. Стиган. Справочник по специальным функциям (М., Наука, 1979).

Редактор Л.В. Шаронова 


\section{Calculation of Coulomb acceptor resonant states in zero-gap semiconductors}

M.S. Zholudev ${ }^{\mathbf{1 , 2}}$, V.V. Rumyantsev ${ }^{\mathbf{1 , 2}, \text { S.V. Morozov }}{ }^{\mathbf{1 , 2}}$

${ }^{1}$ Institute for Physics of Microstructures,

Russian Academy of Sciences,

603950 Nizhny Novgorod, Russia

${ }^{2}$ Lobachevsky State University of Nizhny Novgorod,

603950 Nizhny Novgorod, Russia

Abstract Wavefunctions of electron have been calculated for conduction band of zero-gap semiconductor with Coulomb acceptor. Spherically symmetric Luttinger model was used. The resonant state energies have been calculated as functions of electron and hole mass ratio. 